
Not for reproduction, distribution or commercial use.

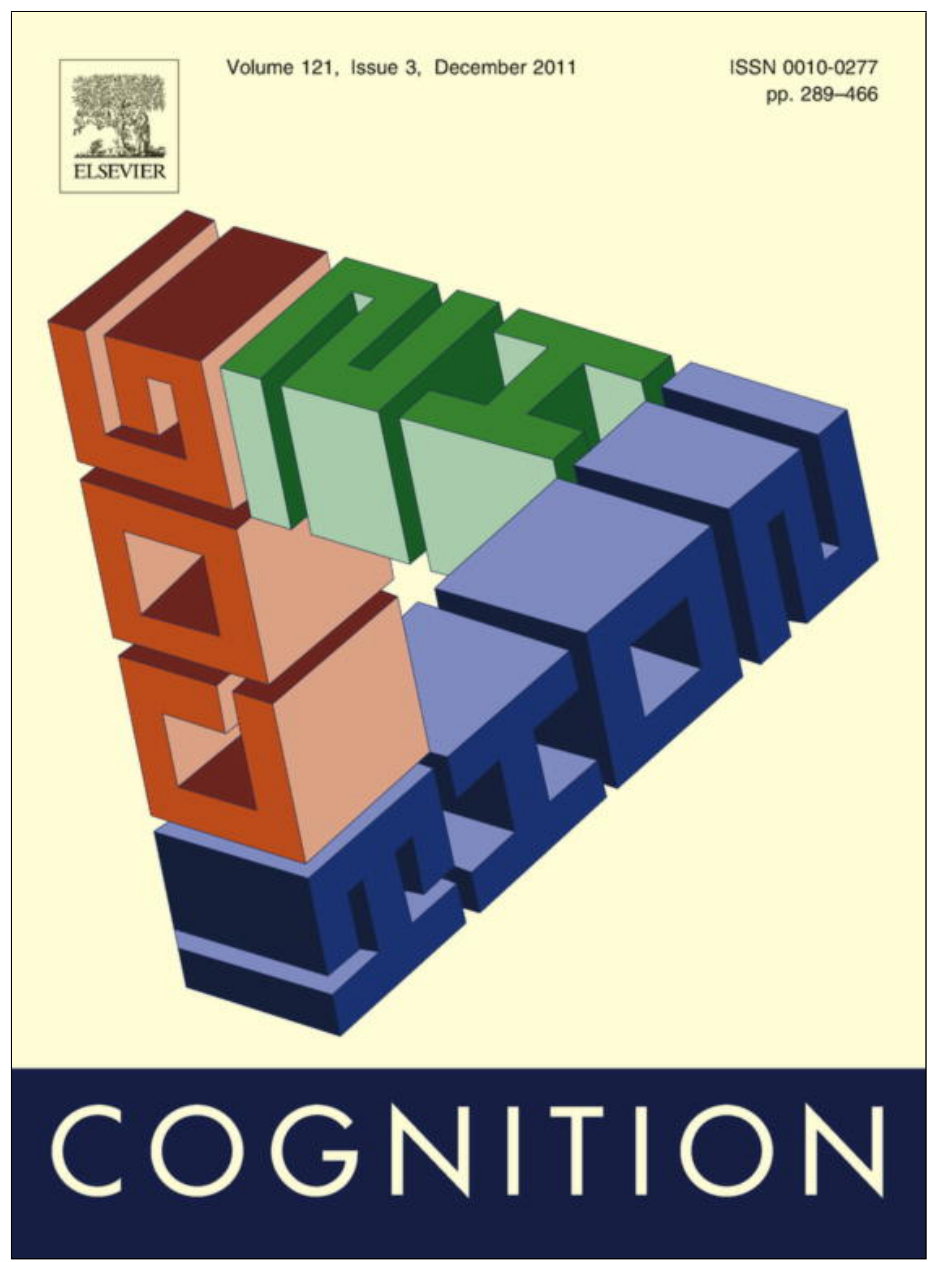

This article appeared in a journal published by Elsevier. The attached copy is furnished to the author for internal non-commercial research and education use, including for instruction at the authors institution and sharing with colleagues.

Other uses, including reproduction and distribution, or selling or licensing copies, or posting to personal, institutional or third party websites are prohibited.

In most cases authors are permitted to post their version of the article (e.g. in Word or Tex form) to their personal website or institutional repository. Authors requiring further information regarding Elsevier's archiving and manuscript policies are encouraged to visit:

http://www.elsevier.com/copyright 
Brief article

\title{
Contextual modulation of reading rate for direct versus indirect speech quotations
}

\author{
Bo Yao*, Christoph Scheepers* \\ Institute of Neuroscience and Psychology, University of Glasgow, Scotland, UK
}

\section{A R T I C L E I N F O}

\section{Article history:}

Received 28 February 2011

Revised 20 July 2011

Accepted 8 August 2011

Available online 8 September 2011

\section{Keywords:}

Direct speech

Indirect speech

Perceptual simulation

Reading

\begin{abstract}
A B S T R A C T
In human communication, direct speech (e.g., Mary said: "I'm hungry") is perceived to be more vivid than indirect speech (e.g., Mary said [that] she was hungry). However, the processing consequences of this distinction are largely unclear. In two experiments, participants were asked to either orally (Experiment 1) or silently (Experiment 2, eye-tracking) read written stories that contained either a direct speech or an indirect speech quotation. The context preceding those quotations described a situation that implied either a fastspeaking or a slow-speaking quoted protagonist. It was found that this context manipulation affected reading rates (in both oral and silent reading) for direct speech quotations, but not for indirect speech quotations. This suggests that readers are more likely to engage in perceptual simulations of the reported speech act when reading direct speech as opposed to meaning-equivalent indirect speech quotations, as part of a more vivid representation of the former.
\end{abstract}

(c) 2011 Elsevier B.V. All rights reserved.

\section{Introduction}

Direct speech quotations (e.g., Mary said: "I'm hungry") are usually produced and perceived as being more vivid and perceptually engaging than indirect speech quotations (e.g., Mary said [that] she was hungry). An important pragmatic function of direct speech is to provide a demonstration to depict a reported speech act whereas indirect speech offers a mere description of what was said (Clark \& Gerrig, 1990). Not only has this vividness distinction been observed by linguists (e.g., Tannen, 1986, 1989), it has also been empirically supported by Wade and Clark (1993), who showed that in reporting previously overheard dialogues, speakers are more likely to employ direct rather than indirect speech when instructed to entertain (as opposed to just inform) a listener.

\footnotetext{
* Corresponding authors. Address: Institute of Neuroscience and Psychology, 58 Hillhead St., Glasgow G12 8QB, UK. Tel.: +44 1413303606.

E-mail addresses: b.yao@psy.gla.ac.uk (B. Yao), Christoph.Scheepers @glasgow.ac.uk (C. Scheepers).
}

However, little research has addressed the question of how this vividness distinction is represented during written language processing. Although many of us may share the intuition of an "inner voice", particularly when reading direct speech quotations, objective confirmations of such experiences are rather sparse. This is surprising because recent embodied cognition theories propose that language comprehension is grounded in perceptual simulation (i.e. mental re-enactment) of perceptual states and actions (Barsalou, 1999, 2008). Given that the use of direct speech is primarily grounded in vivid demonstrations, such theories would predict that during reading, direct-speech quotations are more likely to trigger enriched perceptual simulations (or spontaneous imagery) of reported speech acts than indirect speech quotations.

One important aspect of the more enriched representation of direct speech concerns the reported speaker's voice. A recent study by Yao, Belin, and Scheepers (in press) found that silent readers are more likely to engage in mental simulations of voice in response to direct speech quotations. They combined fMRI with eye-tracking to measure brain activity while participants were silently reading 
stories containing either direct speech quotations or meaning-equivalent indirect speech quotations. They found that for direct speech quotations, there was significantly more 'top-down' activity in voice-selective areas of the auditory cortex (cf. Belin, Zatorre, Lafaille, Ahad, \& Pike, 2000) than for indirect speech quotations. This suggests that readers are more likely to mentally simulate, or spontaneously imagine, aspects of the quoted speaker's voice during silent reading of direct speech.

The present paper addresses one particular aspect (out of potentially many) of the purported "inner voice" experience during reading of quotations, namely the contextually implied rate of the quoted speech. Specifically, we will examine whether readers adjust their reading rates in accordance with the contextually implied speech rate of the quoted speaker during both oral and silent reading of direct versus indirect speech quotations. By focusing on potential behavioural consequences of voice simulation during reading, the present studies represent an important extension of the brain-imaging research reported in Yao et al. (in press).

Indeed, Alexander and Nygaard (2008) recently demonstrated that reading rates are modulated by auditory imagery. In this study, participants were exposed to pre-recorded voices of either fast or slow speakers. In subsequent reading trials, participants were prompted to imagine those speakers as authors of the given reading materials. It was found that both oral and silent reading rates were faster when participants imagined previously heard fast speakers as authors.

Although related, the present experiments are somewhat different. First, Alexander and Nygaard (2008) did not investigate any influences of quoting style (direct versus indirect speech), which are of paramount interest in our investigations. Second, their experimental setup may have explicitly encouraged auditory imagery during reading, focusing on how the auditory memory of a specific talker's speaking rate modulates reading rates. In contrast, our experiments will examine how linguistic context (implying either a fast-speaking or a slow-speaking quoted protagonist) modulates participants' reading rates during oral and silent reading of quotations. Just as in Yao et al. (in press), participants in our experiments will neither be exposed to auditory samples of specific speakers before reading, nor be instructed or encouraged to 'act out' or imagine voices. Moreover, the reading materials will only contain fictitious, unfamiliar speakers. Thus, our experiments are designed to tap into more automatic voice simulation processes in response to written quotations. Inspired by Alexander and Nygaard (2008), we assume that such automatic voice simulations should be measurable in modulations of oral and silent reading rates, and informed by Yao et al. (in press), we predict that these reading rate adjustments should be stronger for direct speech quotations than for meaning-equivalent indirect speech quotations.

In the following experiments, participants either orally (Experiment 1) or silently (Experiment 2) read short fictitious stories that contained either direct or indirect speech quotations. The quotations were embedded in linguistic contexts describing either fast- or slow-speaking quoted protagonists. Participants' oral and silent reading rates were measured via audio recordings and eye-tracking, respectively.

\section{Experiment 1}

\subsection{Participants}

Twenty native English speakers with no reported reading impairments participated in exchange for $£ 2$. A typical session lasted about $20 \mathrm{~min}$.

\subsection{Stimuli}

Twenty-four quadruples of short fictitious stories were prepared as reading materials (see Appendix 1 in the Supplementary File to this paper). The stories contained either a direct speech (1a, 2a) or an indirect speech quotation (1b, 2b) from a fictitious main protagonist in the story (Quoting Style). Across items, different protagonist names were used. A second variable (Context) was independently manipulated such that the quotations were preceded by a context that described either a fast-speaking (1) or slow-speaking (2) quoted protagonist. Crucially, critical quotation passages within each item (bold texts) were identical between the fast- and slow-speaking Context conditions, and nearly the same between the direct and indirect speech Quoting Style conditions. The quotations were always followed by at least one additional sentence.

(1) It was a typical British day, rainy and gloomy. Sixteen year-old pianist Bobby was going to play in the quarter-finals of a local talent competition. He was extremely nervous before his performance.

a. His mother encouraged him but he was all shaking and said: "No! I can't do it! This is the end of the journey because it is unlikely that I will make it this time."

b. His mother encouraged him but he was all shaking and said that he couldn't do it and that it was the end of the journey because it was unlikely that he would make it this time.

His mother tried to calm him down, saying that it's not the winning that counts, but the taking part.

(2) It was a typical British day, rainy and gloomy. At Glasgow Royal Infirmary, an old man was dying, and too weak to sit up. His family members were sitting around the bed, feeling sad. He wanted to say something, so his daughter placed a cushion under his head.

a. Slowly, he looked around and said: "I'm grateful you're all here. This is the end of the journey because it is unlikely that I will make it this time."

b. Slowly, he looked around and said that he was grateful for their coming and that it was the end of the journey because it was unlikely that he would make it this time.

Then he closed his eyes and everyone burst into tears. 
Four stimulus-lists with counterbalanced item-condition combinations (6 stories per condition per list) were constructed and word-processed into four versions of written scripts. The order of the stories per script was randomized. Each script was randomly assigned to five participants.

\subsection{Procedure}

The experiment was conducted in a sound attenuated room. Participants were instructed to read out the stories from the script in one go and as naturally and fluently as possible. The instructions did not explicitly encourage participants to vocally 'act out' the stories during reading.

After setting up the microphone and digital voice recorder, the experimenter sealed the room and the participant started reading the stories aloud while audio recordings were taken. These were saved as wave-files for later analysis.

\subsection{Data analysis}

All recordings were listened to individually by the experimenter. Around 5\% of the trials were excluded from analysis because of word repetition or substitution errors. The remaining valid recordings were digitally visualized (in millisecond resolution) using GoldWave 4.26. For each trial, the temporal onsets and offsets of the critical quotation passages were identified. Next, oral reading rates (in numbers of syllables per second) for the critical quotations were calculated and submitted to 2 [Quoting Style: direct speech versus indirect speech] $\times 2$ [Context: fast versus slow] ANOVAs with subject $\left(F_{1}\right)$ and item $\left(F_{2}\right)$ as random factors.

\subsection{Results and discussion}

Apart from a Context main effect by subjects $\left(F_{1}(1,19)=4.74, p<.05, F_{2}(1,23)=1.68, p>.2\right)$ there was a significant Context $\times$ Quoting Style interaction $\left(F_{1}(1,19)=\right.$ $\left.8.90, p<.01 ; F_{2}(1,23)=6.40, p<.02\right)$. Exploring the latter, it was found that direct speech quotations were read out significantly faster when the context implied a fast-speaking rather than a slow-speaking quoted protagonist. By contrast, no such contextual modulation of oral reading rate was found for indirect speech quotations (Table 1). This suggests that participants engaged in spontaneous vocal reenactments of the reported speech act when reading aloud direct rather than indirect speech quotations, by adjusting their oral reading rates to the speech rates that were contextually implied. Importantly, the results cannot be explained by differences in length or morphological features (tense, person, etc.) between the two Quoting Style conditions because the interaction involved differential effects of Context within each Quoting Style condition.

\section{Experiment 2}

Experiment 2 was an eye-tracking study using the same experimental items as Experiment 1. The question was whether the results from Experiment 1 would replicate if participants were reading the materials silently for comprehension.

\subsection{Participants}

Fifty-two native English speakers with no reported reading impairments participated in exchange for $£ 3$. A typical session lasted $30 \mathrm{~min}$. Four participants were excluded from analysis due to poor eye-tracking (3 participants) or less than $75 \%$ answering accuracy (1 participant), leaving 48 participants for analysis.

\subsection{Stimuli}

The critical stories (24 items) were identical to those in Experiment 1 . They were mixed with 24 filler stories, which contained no direct or indirect speech quotations to conceal the purpose of the study. Experimental and filler items spanned 4-7 lines on screen. Although it was impossible to keep the formatting absolutely identical, care was taken to ensure that the critical quotations in the four versions per item spanned roughly the same screen areas. Content-related questions were also prepared to encourage reading for comprehension.

\subsection{Procedure}

The experiment was conducted using a SR-Research EyeLink 1000 desk-mounted eye-tracker running at $1000 \mathrm{~Hz}$ sampling rate. Stimulus presentation was controlled by software developed at the University of Massachusetts (EyeTrack 0.7.1). Participants were seated about $70 \mathrm{~cm}$ from a $21 \mathrm{in}$. CRT display running at $100 \mathrm{~Hz}$ refresh rate in $1280 \times 960$ pixel resolution. Three characters equalled about one degree of visual angle. Materials were presented in an 18 pts font printed in black on a light grey background. Line spacing was set to 60 pts such that fixation locations could unambiguously be mapped onto a corresponding line of text. Viewing was binocular, but only the participant's dominant eye was tracked (as determined by a simple parallax test). A chin rest was used to keep viewing distance constant and to prevent strong head movements during reading. Button responses were collected using a hand-held Microsoft USB game pad.

Each participant was assigned to one of four lists containing different versions of experimental items, pseudorandomly interspersed with the filler stories. There were always two fillers at the beginning. At the start of the experiment, the standard EyeLink calibration and validation procedure was performed in which participants had to look at nine fixation targets in random succession. Calibration and validation were repeated during the experiment if the experimenter noticed a decline in measurement accuracy.

Each trial started with the presentation of a central fixation dot for drift-correction, followed by a small rectangle in the same location as the first character of the upcoming text display. A fixation for at least $200 \mathrm{~ms}$ on this rectangle triggered the presentation of the text, so that reading always started in the first character position. Participants were instructed to read the stories carefully and to press 
Table 1

Oral reading rates (in syllables per second, with standard deviations in brackets for the critical quotations in Experiment 1. Also shown are results from 2-tailed paired sample $t$-tests examining the simple effects of Context and Quoting Style.

\begin{tabular}{|c|c|c|c|c|c|c|c|c|}
\hline & & & \multirow[t]{3}{*}{ Direct speech } & \multirow[t]{3}{*}{ Indirect speech } & \multicolumn{4}{|c|}{ Quoting style contrast } \\
\hline & & & & & \multicolumn{2}{|c|}{ By subjects } & \multicolumn{2}{|c|}{ By items } \\
\hline & & & & & $t(19)$ & $p$ & $t(23)$ & $p$ \\
\hline Fast-speaking con & & & $6.17(1.0)$ & $5.85(0.9)$ & 3.02 & 0.007 & 2.41 & .03 \\
\hline Slow-speaking co & & & $5.88(0.9)$ & $5.93(1.0)$ & -0.37 & .71 & -0.46 & .65 \\
\hline \multirow[t]{4}{*}{ Context contrast } & By subjects & $t(19)$ & 3.65 & -0.37 & & & & \\
\hline & & $p$ & .002 & .71 & & & & \\
\hline & By items & $t(23)$ & 2.77 & -1.13 & & & & \\
\hline & & $p$ & .01 & .27 & & & & \\
\hline
\end{tabular}

Note: Significant contrasts are highlighted in bold.

a button when they had finished reading. A simple yes/no comprehension question was then presented, which participants had to answer using either the left ('yes') or the right ('no') response button. Answering the question trigged the presentation of the next trial.

\subsection{Data analysis}

Fixation coordinates were mapped onto character positions using EyeDoctor 0.6.5. Fixations below $80 \mathrm{~ms}$ were pooled with temporally adjacent fixations if the latter were within half a degree of visual angle. Fixations on the critical quotation regions were summarized in terms of (i) go-past time (also known as regression path duration), i.e. the time from fixating the critical region for the first time until a subsequent region is fixated, and (ii) no-regressions go-past time (the same as before, but excluding trials where readers regressed back to an earlier region after initial reading of the critical region).

Outlier trials were removed in two steps. First, we excluded trials where the initial reading of the critical region was not fluent, operationally defined by a Fixation Density Disparity greater than two (see Appendix 2 in the Supplementary File). Next, trials with a go-past time of more than 3 SD above an item's condition mean were removed. In total, 74 trials (6.4\%, equally distributed across conditions) were excluded as a result of these two outlier definitions. Data from the remaining 1078 trials were submitted to $2 \times 2$ ANOVAs by participants and items.

\subsection{Results and discussion}

There was a significant Context $\times$ Quoting Style interaction in go-past time $\left(F_{1}(1,47)=22.79, \quad p<.001\right.$, $\left.F_{2}(1,23)=6.41, p<.02\right)$ : Direct speech quotations were read significantly faster when the context implied a fastspeaking rather than a slow-speaking protagonist, while no such Context contrast was found for indirect speech quotations (Table 2). In no-regressions go-past time (excluding $16 \%$ of trials where readers regressed back to an earlier region) the same Context $\times$ Quoting Style interaction emerged $\left(F_{1}(1,47)=14.90, p<001, F_{2}(1,23)=4.91, p<.04\right.$, Table 3$)$. The latter confirms that the go-past time effects in Table 2 were not driven by re-inspections of earlier regions.

The by-subject $t$-tests in Tables 2 and 3 also suggest a 'reversed' (fast > slow) simple effect of Context in the indi- rect speech condition. Closer inspection revealed that this contrast was due to only three stimuli (items 1,23 , and 24 in Appendix 1 of the Supplementary File). After removing those stimuli from analysis, the Context $\times$ Quoting Style interaction remained significant (go-past time: $F_{1}(1,47)=7.50, p<.01, F_{2}(1,20)=4.65, p<.05$; no-regressions go-past time: $F_{1}(1,47)=6.00, p<.01, F_{2}(1,20)=3.90$, $p=.062)$, but crucially, the Context effect in the indirect speech condition disappeared (Tables 4 and 5). It is therefore hard to interpret the latter as anything other than item-specific idiosyncrasy. By contrast, the Context-effect in the direct speech condition (fast < slow) robustly showed up in all analyses.

In conclusion, not only oral readers (Experiment 1), but also silent readers adjust their reading rates to the contextually implied speech rate when reading direct speech as opposed to indirect speech quotations. Again, the observed interactions cannot be explained by differences in length and/or morphology between the two Quoting Style conditions. Additional analyses of numbers and average durations of go-past-fixations (Appendix 3 of the Supplementary File) revealed that the reported go-past time effects were mainly carried by fewer fixations being made in the fast-speaking conditions. Average durations of gopast fixations did not seem to be affected much by the experimental manipulations.

Finally, we also performed by-item correlation analyses to evaluate the consistency of the results between the two experiments. Indeed, the raw oral reading times (in $\mathrm{ms}$ ) from Experiment 1 correlated well with the silent reading data from Experiment $2(r(94)=.86, p<.001){ }^{1}$ Given that these correlations are partly due to length effects (longer quotations take longer to read both orally and silently), we also performed multiple regression analyses with silent reading times (Experiment 2 ) as criterion variables, and both length (in numbers of words) and raw oral reading times (Experiment 1) as simultaneous predictors. These analyses obtained partial correlations of $r(93)=.56(p<.001)$, between Experiment 1 and 2, which means that the two experiments obtained very consistent by-item results even when length effects were partialled out.

\footnotetext{
${ }^{1}$ We considered both go-past and no-regressions go past times in these correlation analyses. Rounded to the second decimal, the $r$-values were identical.
} 
Table 2

Go-past times (in ms, with standard deviations in brackets) for the critical quotations in Experiment 2 (silent reading). Also shown are results from 2-tailed paired sample $t$-tests examining the simple effects of Context and Quoting Style.

\begin{tabular}{|c|c|c|c|c|c|c|c|c|}
\hline & & & \multirow[t]{3}{*}{ Direct speech } & \multirow[t]{3}{*}{ Indirect speech } & \multicolumn{4}{|c|}{ Quoting style contrast } \\
\hline & & & & & \multicolumn{2}{|c|}{ By subjects } & \multicolumn{2}{|c|}{ By items } \\
\hline & & & & & $t(47)$ & $p$ & $t(23)$ & $p$ \\
\hline Fast-speaking con & & & $1857(743)$ & $2056(885)$ & -3.17 & .003 & -2.56 & .02 \\
\hline Slow-speaking co & & & $2011(830)$ & $1942(896)$ & 0.50 & .62 & 0.29 & .78 \\
\hline \multirow[t]{4}{*}{ Context contrast } & By subjects & $t(47)$ & -3.50 & 1.84 & & & & \\
\hline & & $P$ & .001 & .07 & & & & \\
\hline & By items & $t(23)$ & -2.32 & 1.43 & & & & \\
\hline & & $P$ & .03 & .17 & & & & \\
\hline
\end{tabular}

Note: Significant contrasts are highlighted in bold.

Table 3

No-regressions go-past times (in ms, with standard deviations in brackets) for the critical quotations in Experiment 2 (silent reading). Also shown are results from 2-tailed paired sample t-tests examining the simple effects of Context and Quoting Style.

\begin{tabular}{|c|c|c|c|c|c|c|c|c|}
\hline & & & \multirow[t]{3}{*}{ Direct speech } & \multirow[t]{3}{*}{ Indirect speech } & \multicolumn{4}{|c|}{ Quoting style contrast } \\
\hline & & & & & \multicolumn{2}{|c|}{ By subjects } & \multicolumn{2}{|c|}{ By items } \\
\hline & & & & & $t(47)$ & $p$ & $t(23)$ & $p$ \\
\hline Fast-speaking con & & & $1784(693)$ & $1957(853)$ & -3.42 & .001 & -2.43 & .02 \\
\hline Slow-speaking co & & & 1932 (799) & $1845(820)$ & 1.01 & .32 & 0.04 & .97 \\
\hline \multirow[t]{4}{*}{ Context contrast } & By subjects & $t(47)$ & -3.06 & 2.55 & & & & \\
\hline & & $P$ & .004 & .01 & & & & \\
\hline & By items & $t(23)$ & -2.42 & 1.16 & & & & \\
\hline & & $P$ & .02 & .26 & & & & \\
\hline
\end{tabular}

Note: Significant contrasts are highlighted in bold.

Table 4

Go-past times (in ms, with standard deviations in brackets) for the critical quotations in Experiment 2 (silent reading), after removing items 1, 23, and 24 from analysis. Also shown are results from 2-tailed paired sample t-tests examining the simple effects of Context and Quoting Style.

\begin{tabular}{|c|c|c|c|c|c|c|c|c|}
\hline & & & \multirow[t]{3}{*}{ Direct speech } & \multirow[t]{3}{*}{ Indirect speech } & \multicolumn{4}{|c|}{ Quoting style contrast } \\
\hline & & & & & \multicolumn{2}{|c|}{ By subjects } & \multicolumn{2}{|c|}{ By items } \\
\hline & & & & & $t(47)$ & $p$ & $t(20)$ & $p$ \\
\hline Fast-speaking col & & & $1919(752)$ & $2043(897)$ & -1.90 & .06 & -2.13 & .05 \\
\hline Slow-speaking cc & & & $2081(828)$ & 2024 (915) & 0.35 & .73 & 0.63 & .54 \\
\hline \multirow[t]{4}{*}{ Context contrast } & By subjects & $t(47)$ & -3.81 & 0.25 & & & & \\
\hline & & $P$ & .001 & .80 & & & & \\
\hline & By items & $t(20)$ & -2.78 & 0.37 & & & & \\
\hline & & $P$ & .01 & .72 & & & & \\
\hline
\end{tabular}

Note: Significant contrasts are highlighted in bold.

Table 5

No-regressions go-past times (in ms, with standard deviations in brackets) for the critical quotations in Experiment 2 (silent reading), after removing items 1,23 , and 24 from analysis. Also shown are results from 2-tailed paired sample t-tests examining the simple effects of Context and Quoting Style.

\begin{tabular}{|c|c|c|c|c|c|c|c|c|}
\hline & & & \multirow[t]{3}{*}{ Direct speech } & \multirow[t]{3}{*}{ Indirect speech } & \multicolumn{4}{|c|}{ Quoting style contrast } \\
\hline & & & & & \multicolumn{2}{|c|}{ By subjects } & \multicolumn{2}{|c|}{ By items } \\
\hline & & & & & $t(47)$ & $p$ & $t(20)$ & $p$ \\
\hline Fast-speaking con & & & $1843(701)$ & $1941(860)$ & -2.14 & .04 & -2.00 & .06 \\
\hline Slow-speaking co & & & $1996(800)$ & $1904(838)$ & 1.45 & .15 & 0.64 & .53 \\
\hline \multirow[t]{4}{*}{ Context contrast } & By subjects & $t(47)$ & -3.27 & 0.85 & & & & \\
\hline & & $P$ & .002 & .40 & & & & \\
\hline & By items & $t(20)$ & -2.80 & 0.33 & & & & \\
\hline & & $P$ & .01 & .75 & & & & \\
\hline
\end{tabular}

Note: Significant contrasts are highlighted in bold. 


\section{General discussion}

In two experiments, we found that both oral and silent readers adjust their reading rates in accordance with the contextually implied speech rate of a quoted protagonist, but only when quotations employed a direct rather than an indirect speech quotation style. In line with recent fMRI research (Yao et al., in press), the current findings (particularly from Experiment 2) support a hypothesised 'inner voice' experience during silent reading of direct speech quotations; more specifically, we showed that speech rate is an important aspect of this inner voice experience and that mental simulations of voice are not only reflected in brain-activation patterns, but also in behavioural eyemovement patterns.

The present results also extend previous findings by Alexander and Nygaard (2008) who found that pre-exposure to the speech rates of specific speakers influences how fast oral and silent readers would process written stimuli that were supposedly authored by those speakers. Specifically, the present experiments did not focus on this kind of speaker adaptation. Instead, we were interested in how information provided by the linguistic context (implying either a fast-speaking or a slow-speaking quoted protagonist) influences participants' reading rates during oral and silent reading, and importantly, how different quoting styles would affect such contextual modulations of reading rate. Another aspect in which our studies differed from Alexander and Nygaard (2008)'s - apart from using eye-tracking instead of self-paced reading for silent reading - is that our experimental instructions did not explicitly encourage participants to 'act out' or imagine speech during reading (see Introduction). In Experiment 1 (oral reading) participants were just asked to read the stories aloud and in Experiment 2 (silent reading) experimental tasks emphasised reading for comprehension. The observed contextual modulations of reading rate in response to direct speech quotations therefore suggest that, in this type of quotations, readers routinely and automatically activate mental representations that link to how the quoted speaker would sound like (in this case, how fast the quoted protagonist would speak), which in turn have an effect on the rate of information uptake (silent reading) and delivery (oral reading). In this respect, the present results are an important extension of Yao et al. (in press)'s findings, who equally found that silent readers of direct speech quotations routinely and automatically activate voice-related perceptual representations.

Our findings may be interpreted in terms of perceptual simulation, as proposed by embodied cognition theories (e.g., Barsalou, 1999, 2008). Such theories argue that mental representations of language are grounded in perceptual experiences and actions, and that perceptual simulation (i.e. the mental re-enactment of perceptual, motor, and introspective states acquired during experience with the world, body and mind) is an automatic and integral part of language comprehension. Under such a premise, one could argue that accumulated experiences with how direct versus indirect speech quotations are typically used form the basis for differential degrees of perceptual simulation during language comprehension. When speakers employ direct speech, they often mimic or dramatize aspects of the reported speaker's voice in order to depict the reported speech act; indirect speech, by contrast, is typically not used in such a demonstrative fashion (Clark \& Gerrig, 1990). Comprehension of direct speech is therefore more likely to be grounded in the perceptual experience of a vocal demonstration or dramatization of a reported speaker's utterance, and thus more likely to invoke perceptual simulations of the reported speaker's voice. Put differently, a direct-speech reporting style is more likely to be taken as a cue to spontaneously engage in vivid perceptual simulations of the reported speech act than an indirect-speech reporting style.

Of course, there are still a number of open questions which our research cannot answer at present. Some are related to the nature of voice simulation itself. For instance, is the level at which voice simulation operates primarily prosodic, and what kind of processing takes place in conditions where voice simulation is reduced or absent? Other questions relate to eye-movement control in reading. The results from Experiment 2 suggest that high-level factors such as context and quoting style have an impact on basic reading rates. Do these pragmatically induced reading-rate modulations operate at the level of individual word processing or at the level of more 'global' reading parameters? As these questions indicate, further research into this area might substantially deepen our understanding of written language processing as a whole.

In conclusion, our investigations addressed the pragmatics of different quotation styles and their associated mental representations during text comprehension. Our experiments showed that oral and silent readers spontaneously adjust their reading rates to contextually implied speech rates when reading direct speech as opposed to meaning-equivalent indirect speech quotations. These reading-rate adjustments may be interpreted as perceptual simulations of reported speech acts. While previous research on auditory simulation has mostly focused on the processing of sound-related words (e.g., Kellenbach, Brett, \& Patterson, 2001; Kiefer, Sim, Herrnberger, Grothe, \& Hoenig, 2008), the present study and that by Yao et al. (in press) are the first that looked into auditory simulation as a function of pragmatic factors. Our findings provide additional empirical support for a representational distinction between direct and indirect speech during reading, suggesting that perceptual vividness is a key aspect differentiating the two.

\section{Appendix A. Supplementary material}

Supplementary data associated with this article can be found, in the online version, at doi:10.1016/j.cognition. 2011.08.007.

\section{References}

Alexander, J. D., \& Nygaard, L. C. (2008). Reading voices and hearing text: Talker-specific auditory imagery in reading. Journal of Experimental Psychology - Human Perception and Performance, 34(2), 446-459. 
Barsalou, L. W. (1999). Perceptual symbol systems. Behavioral and Brain Sciences, 22(4), 577.

Barsalou, L. W. (2008). Grounded cognition. Annual Review of Psychology, 59, 617-645.

Belin, P., Zatorre, R. J., Lafaille, P., Ahad, P., \& Pike, B. (2000). Voice-selective areas in human auditory cortex. Nature, 403(6767), 309-312.

Clark, H. H., \& Gerrig, R. J. (1990). Quotations as demonstrations. Language, 66(4), 764-805.

Kellenbach, M. L., Brett, M., \& Patterson, K. (2001). Large, colorful, or noisy? Attribute- and modality-specific activations during retrieval of perceptual attribute knowledge. Cognitive Affective and Behavioral Neuroscience, 1(3), 207-221.

Kiefer, M., Sim, E. J., Herrnberger, B., Grothe, J., \& Hoenig, K. (2008). The sound of concepts: Four markers for a link between auditory and conceptual brain systems. Journal of Neuroscience, 28(47), $12224-12230$
Tannen, D. (1986). Introducing constructed dialogue in Greek and American conversational and literary narrative. In F. Coulmas (Ed.), Direct and indirect speech. Berlin; New York: Mouton de Gruyter.

Tannen, D. (1989). "Oh talking voice that is so sweet": Constructing dialogue in conversation. In D. Tannen (Ed.), Talking voices: Repetition, dialogue, and imagery in conversational discourse. Cambridge: Cambridge University Press.

Wade, E., \& Clark, H. H. (1993). Reproduction and demonstration in quotations. Journal of Memory and Language, 32(6), 805-819.

Yao, B., Belin, P., \& Scheepers, C. (in press). Silent reading of direct versus indirect speech activates voice-selective areas in the auditory cortex. Journal of Cognitive Neuroscience 52, doi:10.1162/ jocn_a_00022. 